Supporting information

\title{
Development of a Novel Curing Accelerator-Blowing Agent for Formulating Epoxy Rigid Foam Containing Aminated-Lignin
}

Saeid Nikafshar ${ }^{a}$, Zhen Fang ${ }^{* b, c}$, and Mojgan Nejad*a,d

${ }^{a}$ Department of Forestry, Michigan State University, 480 Wilson Rd, East Lansing, MI 48824

${ }^{\mathrm{b}}$ Department of Biochemistry \& Molecular Biology, Michigan State University, 603 Wilson

Road, East Lansing, Michigan 48824, United States

${ }^{\mathrm{c}}$ Great Lakes Bioenergy Research Center, Michigan State University, 164 Food Safety and Toxicology Building, East Lansing, Michigan, 48824, United States

${ }^{d}$ Chemical Engineering and Materials Science, Michigan State University, 428 S Shaw Ln, East Lansing, MI 48824

KEYWORDS: Amination, Epoxy Foam, Lignin, Bisphenol A, Blowing Agent CORRESPONDING AUTHOR: *Zhen Fang (fangzhe8@msu.edu)

*Mojgan Nejad (nejad@msu.edu)

This supporting information contains two tables (Page S2), seven figures (Page S3-S6), and one video file (Page S7) in six pages. 
Table S1. Spectrophotometer data of original and aminated-lignin

\begin{tabular}{lccc}
\hline Type of lignin & L & a & b \\
\hline Original lignin & $42.2 \pm 0.3$ & $9.5 \pm 0.2$ & $12.5 \pm 0.5$ \\
Aminated-lignin & $56.7 \pm 0.2$ & $4.8 \pm 0.1$ & $14.7 \pm 0.4$
\end{tabular}

Note: "a" value is associated with the difference between red and green; higher "a" corresponds to redder and lower "a" indicates greener. "b" is associated with the difference in yellow and blue; higher "b" implies yellower.

Table S2. Contact angles of water droplets on original and aminated-lignin at different time intervals

\begin{tabular}{|c|c|c|c|c|}
\hline \multirow{2}{*}{ Time (s) } & \multicolumn{2}{|c|}{ Unmodified lignin } & \multicolumn{2}{|c|}{ Aminated-lignin } \\
\hline & Left angle $(\theta)$ & Right angle $(\Theta)$ & Left angle $(\theta)$ & Right angle $(\theta)$ \\
\hline 0 & $117.5 \pm 0.4$ & $110.4 \pm 0.6$ & $115.6 \pm 0.32$ & $127.4 \pm 0.09$ \\
\hline 30 & $101.0 \pm 0.3$ & $97.7 \pm 0.5$ & $115.6 \pm 0.02$ & $127.4 \pm 0.04$ \\
\hline 60 & $66.0 \pm 0.3$ & $66.1 \pm 0.6$ & $115.6 \pm 0.03$ & $127.4 \pm 0.04$ \\
\hline 75 & $39.5 \pm 0.2$ & $37.6 \pm 0.2$ & $115.6 \pm 0.03$ & $127.4 \pm 0.02$ \\
\hline 90 & 0 & $20.5 \pm 0.3$ & $115.6 \pm 0.03$ & $127.4 \pm 0.04$ \\
\hline 120 & 0 & 0 & $115.6 \pm 0.03$ & $127.4 \pm 0.02$ \\
\hline 240 & 0 & 0 & $115.6 \pm 0.03$ & $127.4 \pm 0.02$ \\
\hline
\end{tabular}




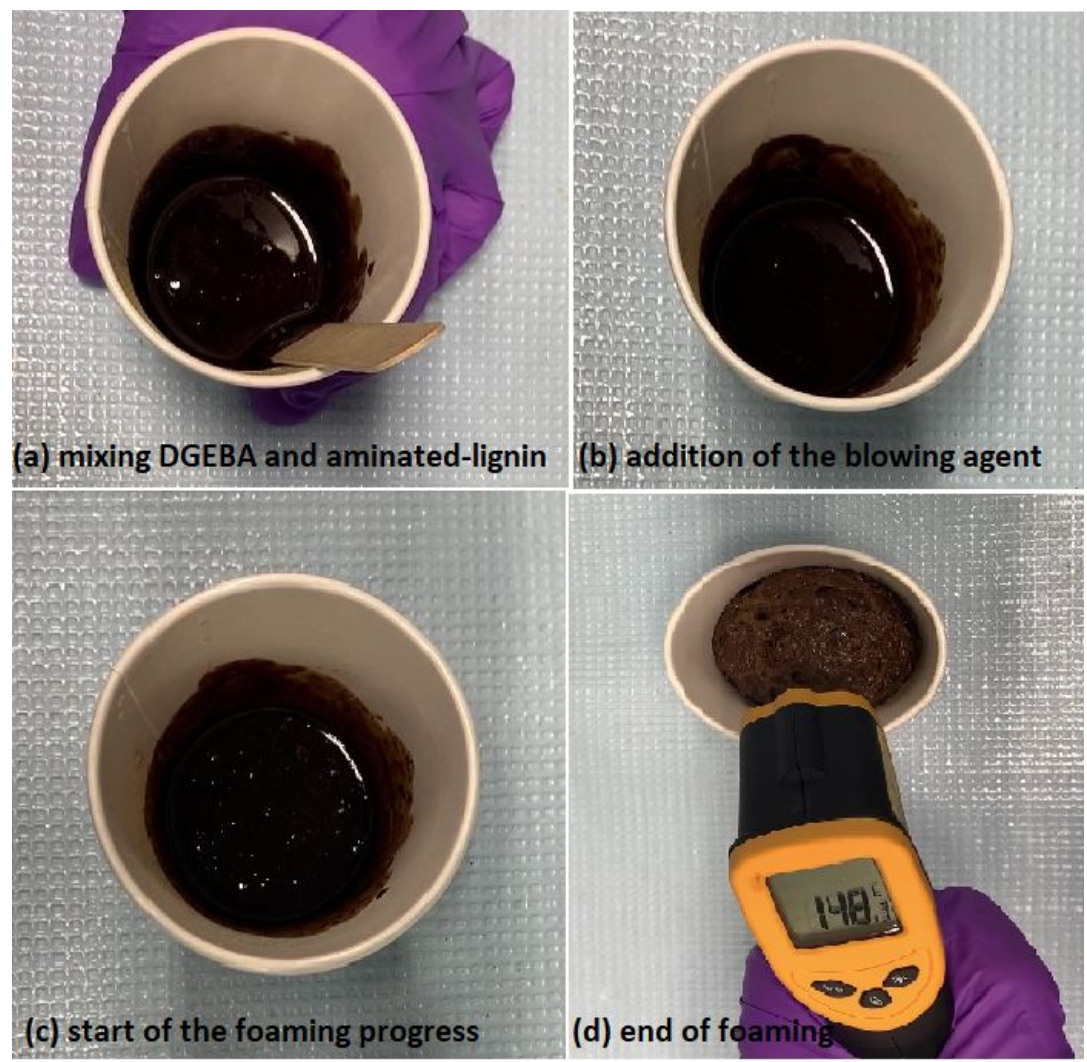

Figure S1. Formulating epoxy foam with aminated-lignin. (a) mixing of DGEBA and aminatedlignin; (b) addition of the blowing agent; (c) start of the foaming progress and (d) at the end of the foam progress 


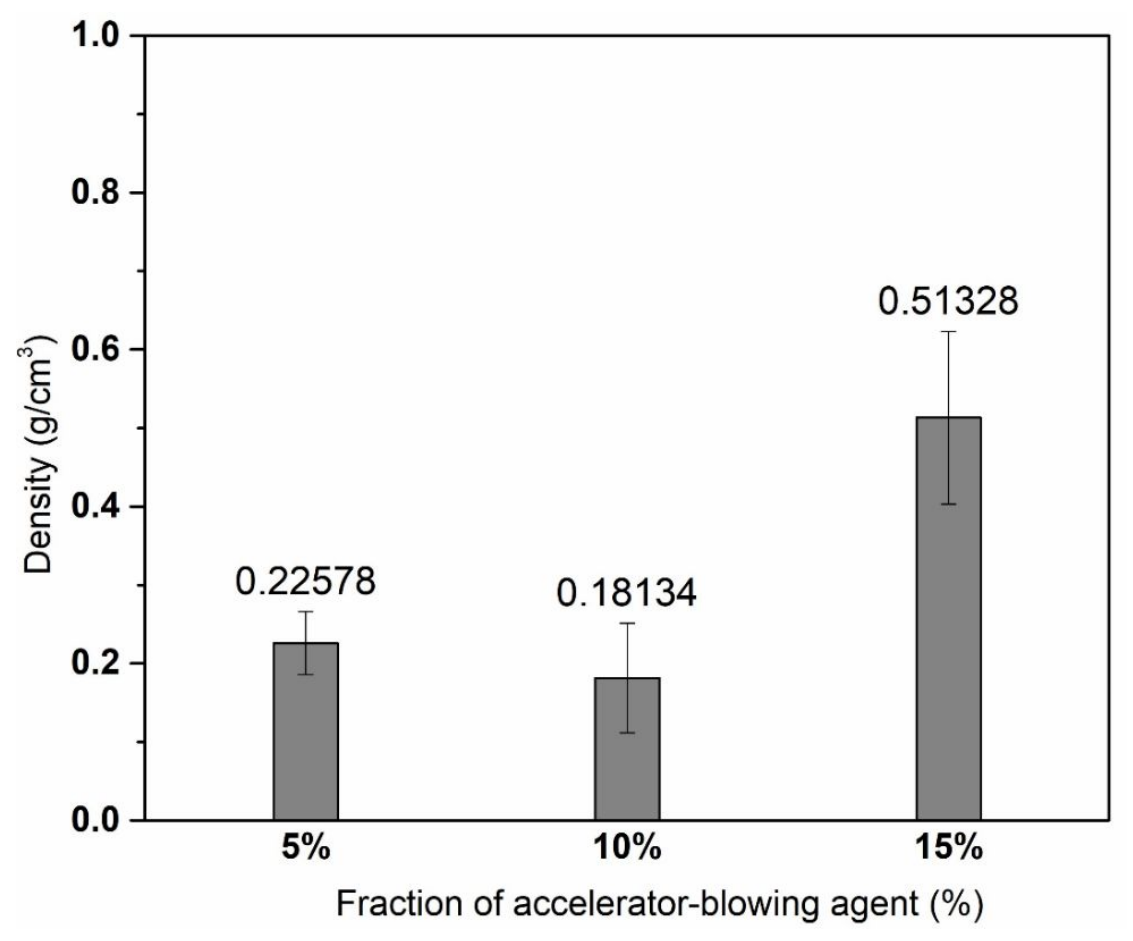

Figure S2. Apparent densities of epoxy foams cured by IPDA (EFIP) with different amounts of blowing agent

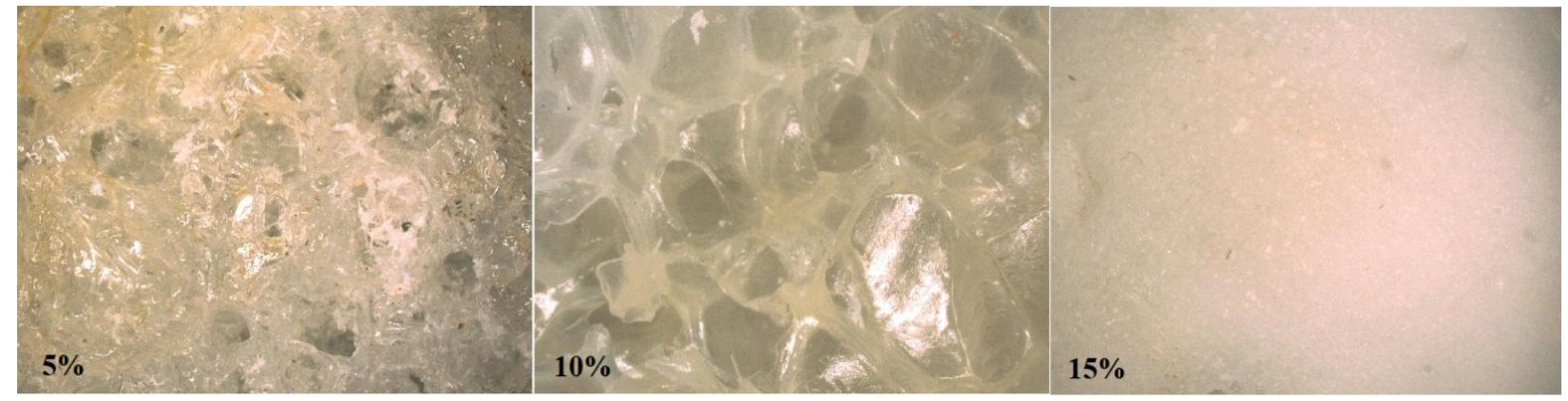

Figure S3. Optical images (30X) of epoxy foams (EFIP) cured by DGEBA/IPDA with different accelerator-blowing agent contents (wt \%) 


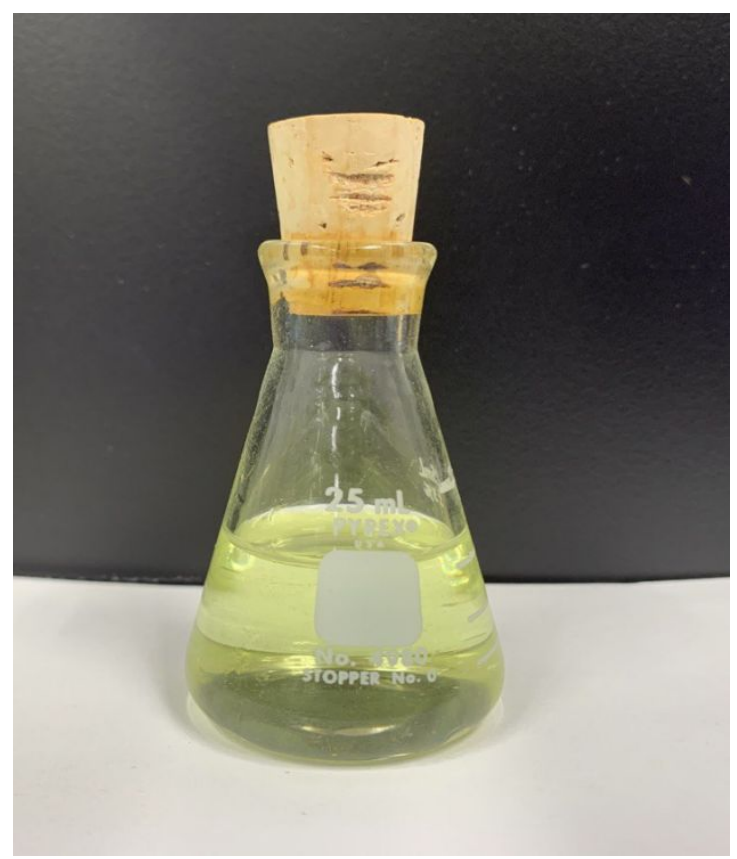

Figure S4. The appearance of the blowing agent solution (pale yellow)

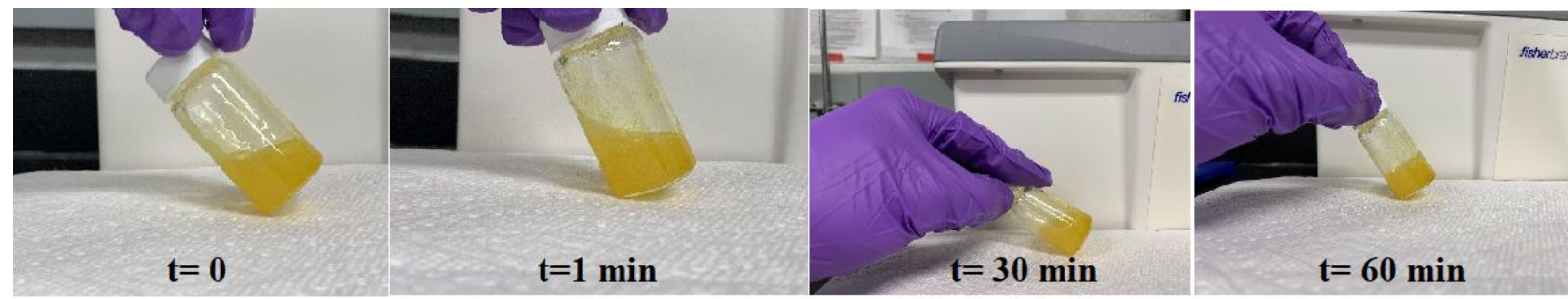

Figure S5. Mixing neat nitric acid (without calcium nitrite) with IPDA and DGEBA 


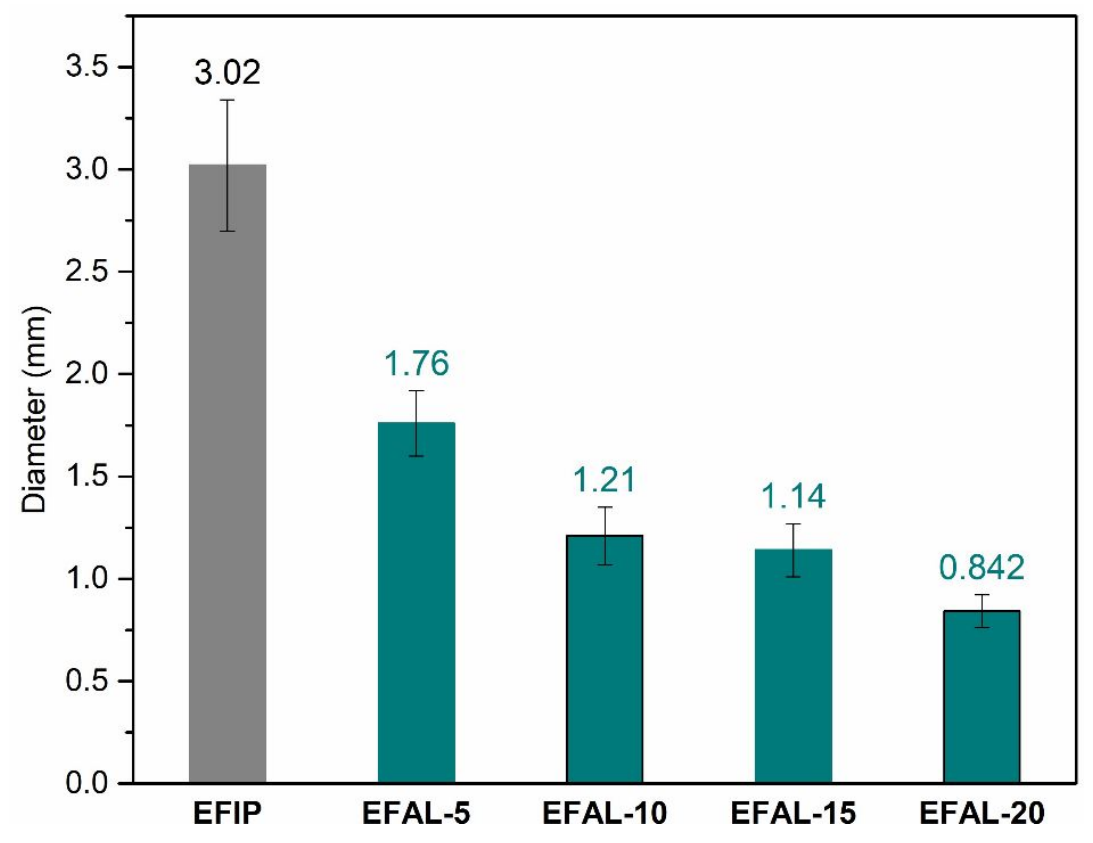

Figure S6. Average cell diameters of different cured epoxy foams

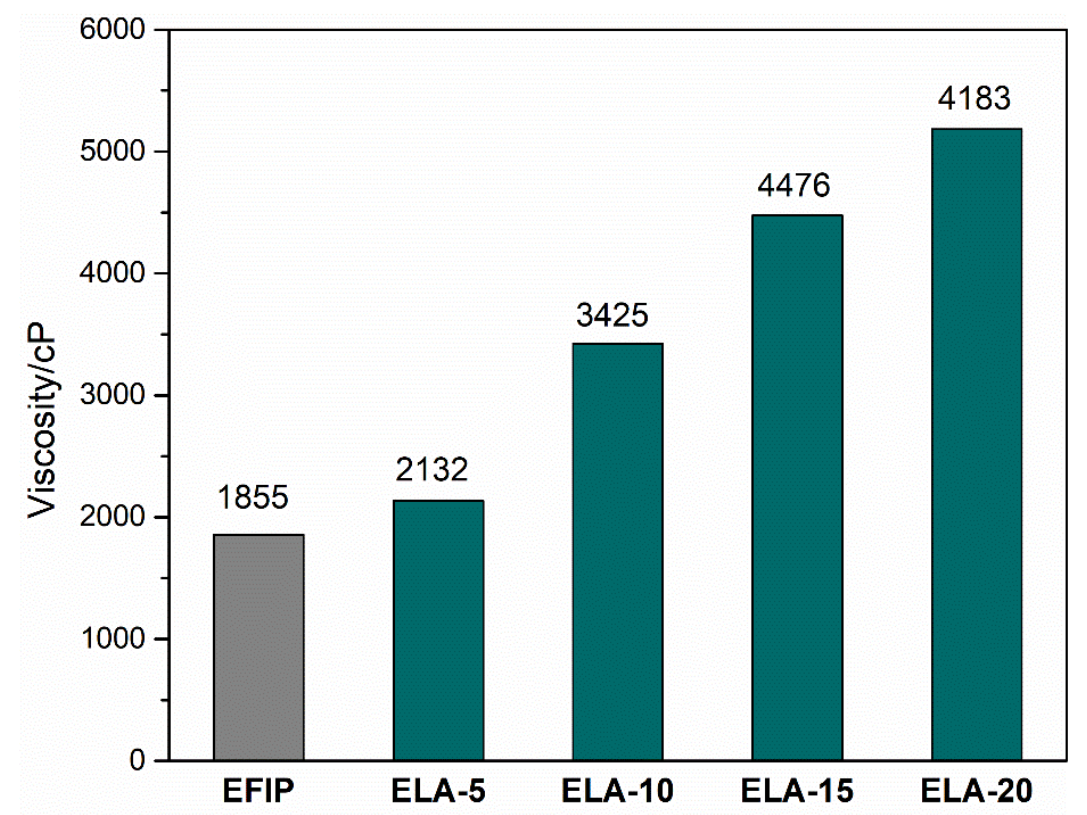

Figure S7. Viscosity of different epoxy foam formulations at $22{ }^{\circ} \mathrm{C}$ 


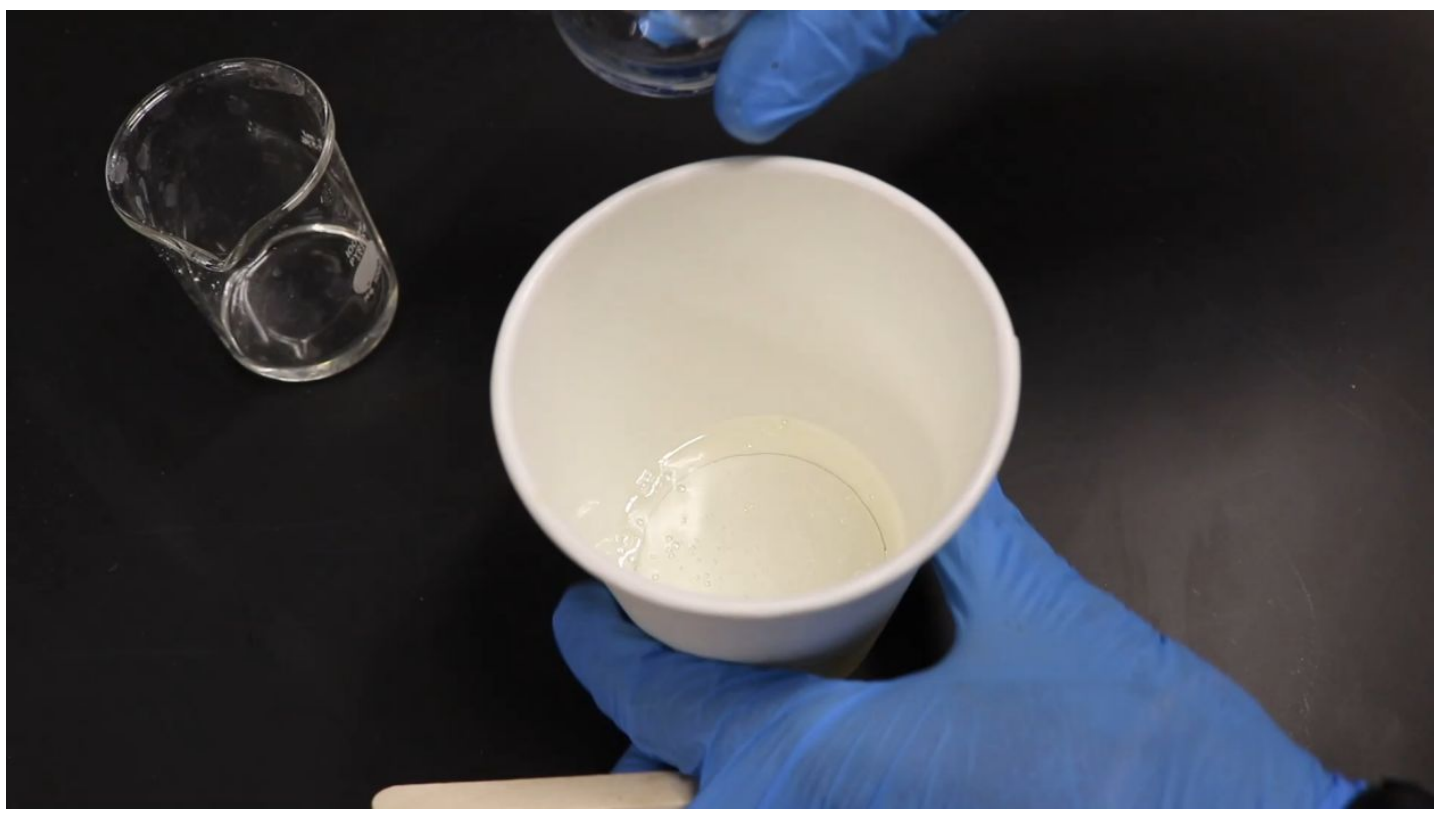

Video S1. Fast-cure of epoxy foam formulated from DGEBA/IPDA in the presence of the accelerator-blowing agent (double left-click to play). Note: The epoxy resin was initially placed in the paper cup, then IPDA, and the blowing agent was added subsequentially. 